\title{
Theodor Kocher und die neurotopographische Diagnostik: Angewandte Forschung mit grundlegendem Ergebnis um 1900
}

Von Ulrich Tröhler

Der Schweizer Nobelpreisträger Theodor Kocher (1841-1917) ist bisher vor allem als Mensch und fachlich als Schilddrüsenchirurg gewürdigt worden ${ }^{1}$. Der ihm 1909 verliehene Nobelpreis «für seine Arbeiten zur Physiologie, Pathologie und Chirurgie der Schilddrüse» ${ }^{2}$ deutet allerdings darauf hin, daß sein chirurgisches Werk auf breiter grundlagenwissenschaftlicher Basis angelegt war. Zudem betraf es neben der Schilddrüse die verschiedensten Gebiete der Medizin. So soll in dieser Arbeit dargestellt werden, wie Kocher um 1895 zur Erarbeitung genauer topographisch-diagnostischer Grundlagen für Eingriffe am zentralen Nervensystem vorging und wie er, sozusagen nebenbei, die Gültigkeit eines wichtigen physiologischen Konzepts, der Dermatome, am Menschen nachwies sowie als erster eine vollständige Tafel der menschlichen Dermatome schuf.

Um 1880 zeigten physiologische Experimente und ergänzende Erfahrungen an Patienten mit Sicherheit, daß gewisse Hirnrindenbezirke Zentren für bestimmte motorische, sensorische und sensitive Funktionen darstellten. Damit kam der Chirurg in die Lage, wegen Reiz- und Lähmungszuständen ganz genau umschriebene Stellen der Hirnrinde aufzusuchen, zumal im Zuge der gleichzeitigen Entwicklung der allgemeinen Chirurgie solche Operationen in den Bereich des Verantwortbar-Möglichen rückten. Eine Schwierigkeit lag allerdings darin, diese krankhaften Veränderungen vorgängig mit einiger Gewißheit zu orten. Ohne die noch unbekannte Radiologie konnte eine Lokalisation nur anhand klinischer Zeichen versucht werden, wofür die Hirnrindenzentren (Foci) ihrerseits die Grundlage darstellten.

Bei Verletzungen und Tumoren des Rückenmarks kam in dieser Hinsicht der Grundvorstellung von nach Rückenmarks-Segmenten gegliederten Bezirken der Hautsensibilität und der Muskelinnervation, den sogenannten sensiblen und motorischen Dermatomen, praktische Bedeutung zu: Von dergestalt als Leitsymptomen aufgefaßten sensorischen und motorischen Ausfällen versprach man sich eine Möglichkeit zur präzisen Ortung spinaler Läsionen. Und nur danach konnte vom wissenschaftlichen Standpunkt eine 


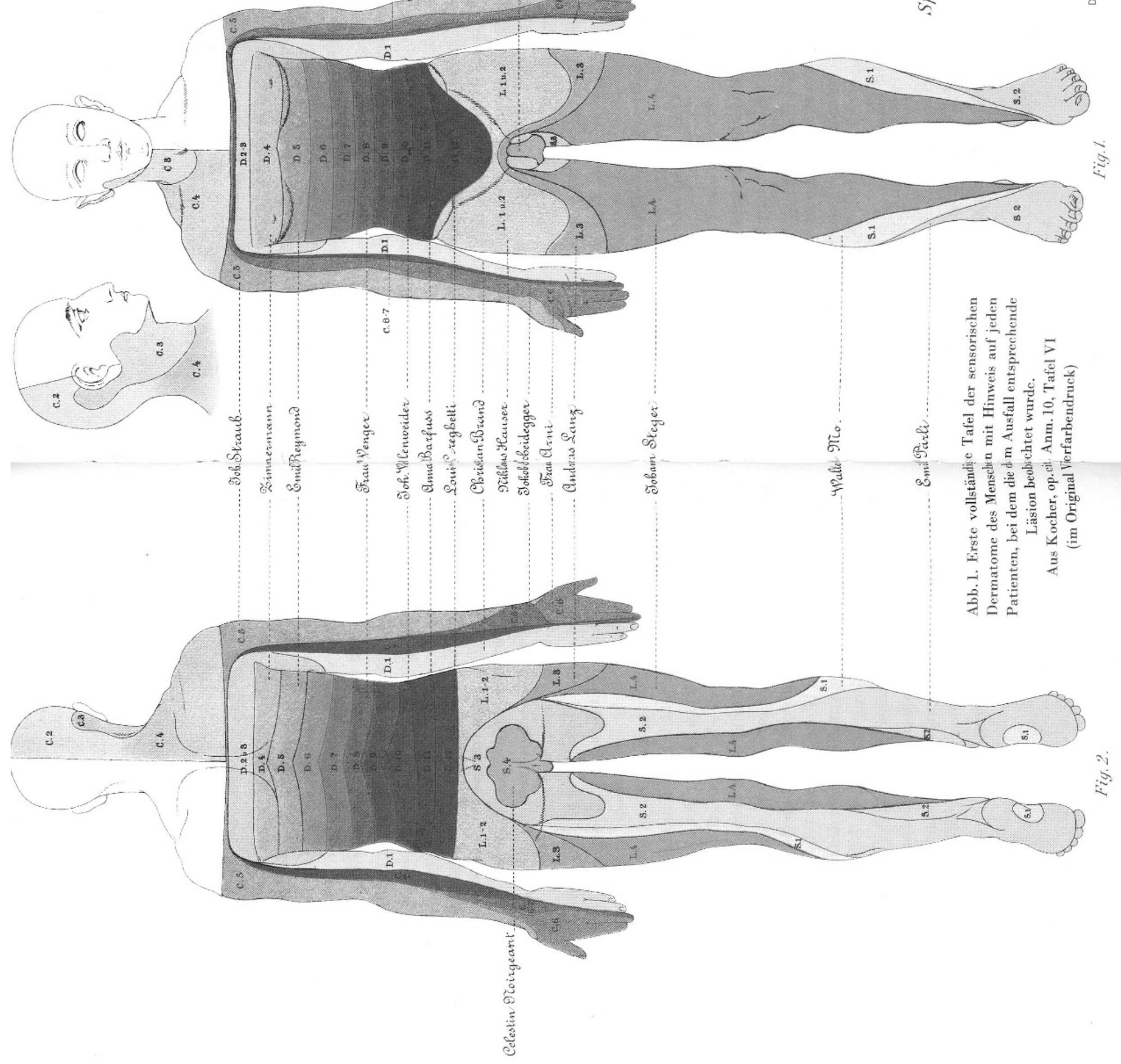


Operation am Rückenmark verantwortet werden, wie sie Kochers Londoner Bekannter Victor Horsley (1857-1916) erstmals im Jahr 1887 erfolgreich versucht hatte ${ }^{3}$. Zu dieser Zeit wurde nun die seit den 1850er Jahren beschränkt bearbeitete, ursprünglich physiologische Frage der Dermatome von verschiedenen Seiten intensiv angegangen: Anatomen verfolgten die periphere Verteilung von Spinalwurzeln, Nervenplexi und einzelnen Fasern mittelst feiner Dissektion. Britische und amerikanische Kliniker untermauerten das Segment-Konzept durch ihre Beobachtungen an Patienten mit Krankheiten oder Verletzungen des Rückenmarks und der peripheren Nerven. Ein anderer Zugang lag in der Erforschung der embryologischen Entwicklung der Zuordnung einzelner Nerven zu bestimmten Muskel- bzw. Hautbezirken.

Einen Überblick über diese frühen Arbeiten gab 1893 der Britische Neurologe Charles S. Sherrington (1857-1952) in der Einleitung zu seinem nunmehr klassischen - Artikel Experiments in the examination of the peripheral distribution of the fibres of the posterior roots of some spinal nerves I ${ }^{4}$. Selbst beschrieb er darin auf der Grundlage von Tierversuchen mit großer Genauigkeit die einzelnen Rückenmarkssegmenten entsprechenden sensiblen Hautzonen an der untern Extremität des Affen. Die Arbeit enthielt auch die heute als «Sherringtonsches Gesetz» bekannte Beobachtung der Überlappung dieser Hautzonen. Allerdings war sich Sherrington bewußt, daß

«das hier angeschnittene Problem dem Tierversuch nur beschränkt zugänglich ist. Es wartet auf eine vollständigere Lösung durch die Gelegenheiten, welche menschliche Krankheit bieten kann, wo die Reaktion eines instruierten Patienten aufgezeichnet und auch menschlich erfragt werden kann». ${ }^{5}$

Für ihn stellten diese sorgfältigen Detailstudien der sensiblen Bahnen denn auch den ersten Schritt in eine andere Richtung dar, nämlich zum Verständnis der Mechanismen der Reflexaktion ${ }^{6}$.

In der Frage der Hautversorgungsgebiete der sensiblen Hinterwurzeln einzelner Rückenmarksnerven versuchten in der Tat nun einzelne Kliniker erfolgreich, eine Übersicht zu gewinnen. So stellte W. Thorburn 1890 und 1893 die verschiedentlich publizierten Einzelfälle mit Angaben über den Verlust der Sensibilität zusammen ${ }^{7}$. Gleichzeitig veröffentlichte Henry Head (1861-1940), ein Freund Sherringtons, zwei unvollständige, aber sehr suggestive Schemata sensibler menschlicher Dermatome. Er hatte sie indirekt bestimmt, unter Benutzung der Verteilung der Hauteruptionen 
verschiedener Herpes-zoster-Fälle und der hyperästhetischen Hautzonen bei gewissen inneren Krankheiten. Dies kam einer klinischen Analyse der Hautverteilung der Hinter-Wurzeln à la Sherrington gleich. Noch heute spricht man ja von den «Headschen Zonen», welche die Segmente des Rückenmarks auf der Basis der Hautirritation veranschaulichen ${ }^{8}$.

Der Kliniker Theodor Kocher hatte seinerseits schon 1885 seine Fälle von Rückenmarkstrauma seit seiner Studentenzeit unter Bezug auf Veränderungen der autonomen Funktion von Blase und Darm sowie von Pulsrate und Körpertemperatur als Dissertation veröffentlichen lassen ${ }^{9}$. Nun machte ihn sein Fakultätskollege, der Physiologe Hugo Kronecker (1839-1914), auf Sherringtons Artikel aufmerksam*10. So nam er auch die erwähnten anglo-amerikanischen klinischen Arbeiten zur Kenntnis und faßte den Entschluß, sich anhand der vorhandenen Krankengeschichten und systematisch zu erforschender neuer Fälle an die Beschreibung aller Dermatome zu machen. Wie aus seinen später veröffentlichten Krankengeschichten ersichtlich ist, begann er jetzt gezielt Befunde an Rückenmarksverletzten im Hinblick auf die aktuell gewordene Frage zu erheben. Wie vor ihm Thorburn und A.Starr in England und nach ihm A. Chipault, P. von Bruns und andere in Frankreich und Deutschland ${ }^{11}$ folgte also Kocher Sherringtons Hinweis, daß solche Fälle eine gute direkte Erforschung der menschlichen Rückenmarksphysiologie erlaubten. Head anerkannte Kochers Beitrag zu Recht, als er 1906 schrieb:

«Unter allen diesen (Klinikern) trat einzig Kocher den Schwierigkeiten entgegen und machte sich daran, die obere Begrenzung (Niveau) der Analgesie in einer großen Zahl von (durch Operation oder Autopsie) verifizierten Rückenmarksläsionen zu bestimmen.» ${ }^{12}$

1939 erinnerte sich Kochers Nachfolger Fritz de Quervain (1868-1940) sehr wohl an den mühseligen Aufwand, der damit verbunden war:

«W as das bedeutet, das weiß noch heute jeder der damaligen Assistenten, der stundenlang an Werk- und Sonntagen die Reflexe der unglücklichen Rückenmarksverletzten beobachten mußte.» ${ }^{13}$

Entsprach diese Gründlichkeit in der klinischen Beobachtung Kocherscher Eigenart, so nicht minder die Umsicht, mit der er aus diesem sorgfältig erhobenen Material, aufgrund weiterer anatomischer und physiologischer Studien, seine Schlüsse zog. Als er mindestens einen genau verfolgten Fall von isolierter Verletzung eines jeden Wirbels und einer jeden Nervenwurzel

* Kocher faßte ibn später als Einleitung zu seiner eigenen Arbeit ausgezeichnet zusammen. 
beisammen hatte, entstanden 1896 in mühsamer Arbeit, einem riesigen Puzzle vergleichbar, anhand der beobachteten sensorischen und motorischen Ausfälle seine Diagramme aller menschlichen Dermatome (Abb.1) und spinalen Motilitätstafeln. Sie waren enthalten in der auch als Monographie erschienenen Arbeit Die Verletzungen der Wirbelsäule, zugleich als Beitrag zur Physiologie des menschlichen Rückenmarks ${ }^{14}$. Wie später Head, bestätigte Kocher darin das Sherringtonsche Gesetz beim Menschen, was Thorburn beispielsweise mißriet. In seiner Einführung zum Schema der sensorischen Dermatome liest man daher:

«Es erschien uns wünschenswert, vom chirurgischen Standpunkt aus, d. h. nach Erfahrungen am Menschen, einmal eine solche Tafel anzulegen, um bei weiteren Beobachtungen die nötigen Korrekturen einzuzeichnen. Denn es ist doch notwendig, die freilich viel exakteren Ergebnisse der experimentellen Forschung am Tier allmählich durch klinische Beobachtungen für den Menschen zu ergänzen, um außer Sensibilitätstafeln, wie wir sie für die Hautversorgung durch die peripheren Nerven besitzen, auch eine solche zu konstruieren, welche man mit Sherrington als Sensibilitätstafeln der spinalen Hautgebiete bezeichnen kann .... ${ }^{15}$

Wie zuvor Starr (1894) ${ }^{16}$ vergaß Kocher neben dieser theoretisch-physiologischen Fragestellung die ursprünglich angestrebte praktisch-klinische Verwertung seiner Feststellungen nicht: die Ortung pathologischer Veränderungen im Rückenmark. Dabei erlaubten die Motilitätstafeln der spinalen Muskelgruppen zusätzlich zu den sensorischen und motorischen Ausfällen noch lokalisatorische Rückschlüsse je nach dem Verhalten der sogenannten tiefen Reflexe und der Sehnenreflexe (Eigen- und Fremdreflexe). In dieser Absicht veröffentlichte Kocher in der Monographie von 1896 denn auch eine neue diagnostische und prognostische Bewertung der Reflexe: Als erster verneinte er nämlich die alte Ansicht, wonach die tiefen Reflexe (d.h. Fremd- und Eigenreflexe) nach Totalsektion des Rückenmarks vollständig verschwänden, was dann zeitgenössische Neurologen als zutreffend anerkannten ${ }^{17}$. Er beschrieb selbst einen neuen dieser tiefen Reflexe ${ }^{18}$.

Rasch stützten sich Chirurgen und Internisten in ihrer praktischen Tätigkeit auf diese nützlichen Angaben, zumal Heads vollständiges, aber nur sensorisches Schema erst 1900 heraus kam ${ }^{19}$. Jüngere Wissenschaftler hatten sie zu beachten.

Wie von Kocher vorausgesehen, wurden hier und dort Korrekturen seiner Angaben notwendig, als eine Anzahl Forscher die Dermatome weiter studierten ${ }^{20}$. Doch bezeichnete noch 1936 der frühere deutsche Neurochirurg Otfrid Foerster (1873-1941), dem es inzwischen gelungen war, die 
meisten menschlichen Dermatome nach Durchtrennung der Nervenwurzeln (Rhizotomie) genau zu beschreiben, Kochers Tafeln als die wohl bekanntesten neben denjenigen von Head ${ }^{21}$. Dies ist sicher teilweise ihrem Eingang in die zeitgenössischen Lehrbücher zuzuschreiben. Das maßgebende amerikanische Werk über den Schmerz enthielt sie ebenso ${ }^{22}$ wie Hermann Sahlis (1856-1918) und William Oslers (1849-1919) mehrfach übersetzte Standardlehrbücher der inneren Medizin, so daß sie darin noch bis anfangs der vierziger Jahre unseres Jahrhunderts unverändert erschienen ${ }^{23}$. Gleicherweise nahm de Quervain Kochers Tafeln in seine Spezielle Chirurgische Diagnostik auf, ein Werk, das von 1907 bis nach dem Zweiten Weltkrieg aufgelegt und in mindestens fünf Sprachen übersetzt wurde ${ }^{24}$.

Die Bedeutung der Kocherschen Arbeit lag einmal in der Schaffung eines in seiner Vollständigkeit und Einheitlichkeit einzig dastehenden Urmaterials, das sich zuverlässig verwerten ließ. Dann schloß Kocher sowohl sensible wie motorische Ausfälle ein, kam zu nützlichen klinischen Folgerungen und faßte seine Beobachtungen auf höchst didaktische Weise in farbigen Tafeln zusammen. Damit gehört ihm die Priorität der ersten kompletten «Kartographie» der menschlichen Dermatome. Die Nützlichkeit derselben in der vor-neuroradiologischen Zeit ist einleuchtend. So kann man diesen Beitrag füglich mit Rudolf Nissen als die «Grundlage der topischen Rückenmarksdiagnostik» bezeichnen ${ }^{25}$.

Was nun das von Kocher benutzte Vorgehen betrifft, so hatte sich die systematische Korrelation klinischer mit pathologisch-anatomischen Beobachtungen, die «méthode anatomo-clinique» der Franzosen, im 18. Jahrhundert entwickelt und in der Pariser Spitalmedizin zu Beginn des 19. Jahrhunderts eine erste Blüte erlebt ${ }^{26}$. Seither behauptete sie sich als eine zuverlässige Forschungsmethode in der Krankheitsbeschreibung ${ }^{27}$. Wir begegnen ihr auch in Kochers vergleichsweise lückenhaftem Versuch der präoperativen Lokalisation pathologischer Veränderungen in einzelnen Hirnbezirken. Dazu nahm er am Ende des 19. Jahrhunderts unter anderen eine 1889 in einer Berner Dissertation vertretene Idee ${ }^{28}$ auf und entwickelte ein eigenes Instrument, das Craniometer (Abb.2).

Dieses unterschied sich im Prinzip dadurch, daß es aufgrund von Verhältniszahlen bei jeder Schädelform Fixpunkte zu bestimmen gestattete, währenddem andere Methoden umgekehrt von bloß palpatorisch festgestellten Fixpunkten ausgingen und mit absoluten Maßen weiter lokalisierten. Die Ergebnisse waren daher, im Gegensatz zu denjenigen Kochers, Abweichungen je nach der Schädelform unterworfen. In einer 
langen Versuchsserie stellte Kocher mit einer Injektionstechnik die Teile der Hirnoberfläche fest, die seinen mit dem Craniometer auf der Kopfoberfläche ermittelten Meßpunkten entsprachen: Nach der Perforation des Schädels an der betreffenden Stelle markierte er sie am Hirn mit Farbstoff und ließ sie nachher durch einen Zeichner festhalten. Dabei stellte er bei wiederholter Beobachtung große Übereinstimmung fest für jene Hauptpunkte, deren Aufsuchung bei Menschen in Frage kam, da ihre Funktion bekannt war ${ }^{29}$.

In letzter Hinsicht mußte er anfänglich auf «die klassischen Untersuchungen Horsleys über die Centren der Hirnrinde beim Affen» zurückgreifen, da man über diejenigen des Menschen noch zu wenig wußte: «Professor Horsley hatte die große Freundlichkeit, uns auf unsere Anfrage hin eine eigenhändige Zeichnung zuzusenden», schrieb Kocher dazu ${ }^{30}$. Später benützte er weitere Affenversuche Sherringtons. Mit der Zeit konnte er auch Beobachtungen anläßlich eigener Hirnoperationen verwerten. Schließlich beruhten seine topographischen Angaben auf den Ergebnissen elektrischer Reizversuche bei Operationen des deutschen Pioniers Fedor Krause (1856-1937), «deren Reproduktion er uns gütigst gestattet hat». ${ }^{31}$

Kochers so mit vielen Mitarbeitern ${ }^{32}$ stets weiter entwickelte Methode war bereits in der ersten Auflage seines Lehrbuchs Chirurgische Operationslehre 1892 enthalten. Sie war nicht die einzige. In seiner Monographie Hirnerschütterung, Hirndruck und chirurgische Eingriffe bei Hirnkrankheiten (1901) gab er einen kritischen Überblick über die seit der Mitte des 19. Jahrhunderts in der Physiologie begonnenen Bemühungen für eine cranio-cerebrale Topographie, die sich, wie bei der spinalen Lokalisation, ausgangs der 1880 er Jahre in der Klinik verstärkt hatten ${ }^{33}$. Wie dort, so war auch hier der Grund ein möglichst beschränktes und daher gezieltes chirurgisches Eingreifen zu ermöglichen. Dies sei nötig, erläuterte er, da die Asepsis zur Entfernung großer Teile des Schädels zu rein diagnostischen Zwecken geführt habe, «Anschauungen, ... welche zum Schaden der Patienten ausgeschlagen haben». Und unmißverständlich warnte er davor, so einen der größten Fortschritte in der Chirurgie zum Anlaß zu Mißbräuchen werden zu lassen:

\footnotetext{
«(Wir dürfen nicht) in den Fehler so vieler Laparatomisten fallen ..., an Stelle einer guten Diagnose eine (solche) sogenannte Explorativincision setzen zu wollen. Diese Ausgeburt der antiseptischen Ära, welche leider noch vielerorts zum Verderben wissenschaftlicher Auffassung bei jungen Ärzten ihre Sanction erhält, sollte dem Bewußtsein weichen, daß nur derjenige, der auf Grund genauester Diagnose eine Operation unternimmt, sich von einem Routinier im Style der alten Bruch- und Steinschneider unterscheidet.» ${ }^{34}$
} 


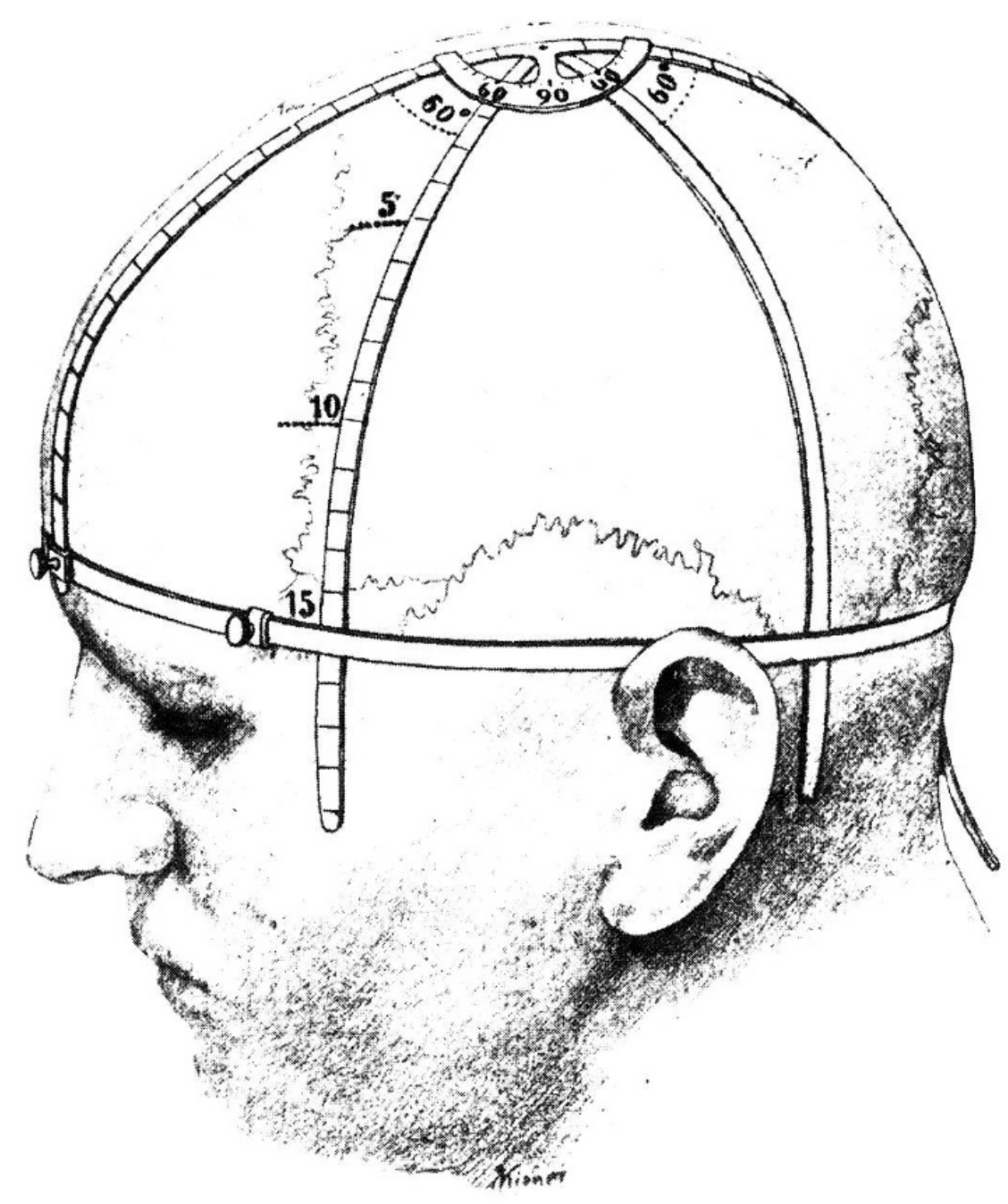

Abb. 2. Letzte Ausführung von Kochers Craniometer mit festem Äquator und Sagittalmeridian und beweglichem Schrägmeridian mit Kreiseinteilung zur Bestimmung der Präzentralfurche und der Sylvischen Furche. Aus Kocher, Anm. 29, 5. Aufl., S. 295 
In dem erwähnten Überblick führte Kocher auch das 1899 «in Anlehnung an unser Vorgehen» veröffentlichte Craniometer seines Zürcher Kollegen Rudolf Ulrich Krönlein (1847-1910) auf. Dieser schwerfälligere Apparat vorab zur Lokalisation der Blutungen aus der Arteria meningea media habe laut einer Dissertation noch bis in die jüngste Zeit seinen Wert behalten ${ }^{35}$. Kochers viel allgemeiner auf Ortung von Hirnrindenstörungen ausgerichtetes Gerät verlor dagegen mit der Neuro-Radiologie von seiner Bedeutung. In seiner Zeit indessen hatte es mit entsprechenden Illustrationen gebührende Beachtung in klassischen Werken wie Harvey Cushings (1869-1939) Surgery of the Head (1908) ${ }^{36}$ und Krauses - auch übersetzter - Chirurgie des Gehirns und Rückenmarks $(1908 ; 1911)^{37}$ gefunden.

Zusammenfassend stellen Kochers cranio-zerebrale Topographie - und vorab seine Dermatom-Tafeln - Meisterstücke klinisch-pathologischer Forschung dar. Sie zeigen, was unmittelbare Beobachtung mit vergleichsweise wenig Hilfsmitteln an einer kleinen Klinik zu leisten vermochte, wenn der Chef den Betrieb mit planmäßigem Sammeln von Krankengeschichten darauf ausrichtete. Sie legen Zeugnis ab über sein auf internationaler Zusammenarbeit fußendes Vorgehen bei der wissenschaftlichen Begründung chirurgischer Eingriffe am Zentralnervensystem. Hervorgerufen durch das Streben nach präoperativer Lokaldiagnose, zeitigte dieses Bemühen für den auf dem Boden der Physiologie stehenden Chirurgen Kocher sozusagen im Nebenschluß grundlegende Erkenntnisse von Bedeutung, gleichermaßen anerkannt von Chirurgen, Internisten und Physiologen. Die Erarbeitung der neurotopographischen Diagnostik war eine wirkliche Tat, die, wiewohl heute vergessen, aus Beweggrund und Ergebnis unsere Hochachtung verdient ${ }^{38}$.

\section{Anmerkungen}

${ }^{1}$ Bonjour, E., Theodor Kocher, 2. Aufl., Bern 1981.

Ackerknecht, E., und H.Buess, Kurze Geschichte der großen Schweizer Ärzte, Bern/Stuttgart/Wien 1975, S.64-67.

Zimmermann, L.M., and I. Veith, Theodor Kocher (1841-1917) and the surgery of the endocrine system, in: Great ideas in the history of surgery, Baltimore 1961, S. 499-518.

Bornhauser, S., Zur Geschichte der Schilddrüsen- und Kropfforschung im 19. Jahrhundert ..., Aarau 1951, S. 53-74, 155-158.

${ }^{2}$ Siehe die Nobelpreisurkunde, wiedergegeben bei Bonjour, op. cit., S.66, Abb. 17.

${ }^{3}$ Morton, L. T., A medical bibliography (Garrison and Morton), Edinburgh 1970, No. 4882. 
${ }^{4}$ Phil. Trans. R. Soc. 184 B (1893) 631-763.

${ }^{5}$ Ibid., zitiert bei Lord Cohen of Birkenhead, Sherrington, physiologist, philosopher and poet, The Sherrington lectures IV, Liverpool 1958, S. 29.

${ }^{6}$ S.Swazey, J.P., Reflexes and motor integration: Sherrington's concept of integrative action, Cambridge Mass. 1969 S. 63-66; Granit, R., Charles Scott Sherrington: an appraisal, London 1966; Liddell, E. G.T., The discovery of reflexes, Oxford 1960, S.123-125.

7 Thorburn, W., The sensory distribution of the spinal nerves, Brain 16 (1893) 355-374, und A contribution to the surgery of the spinal cord, Jacksonian Essay 1890, Manuskript, verfügbar im Royal College of Surgeons, London.

${ }^{8}$ Head, H., On disturbances of sensation with especial references to the pain of visceral disease, Part I «Back», Brain 16 (1893) 1-32; Part II «Head and Neck», ibid. 17 (1894) 339-480; Part III, «Pain in diseases of the Heart and Lungs», ibid. 19 (1896) 153-276.

${ }^{9}$ de Reynier, E., Einige Bemerkungen über 17 Fälle von Wirbelfrakturen, Diss. med. Bern 1885.

${ }^{10}$ Kocher, T., Die Verletzungen der Wirbelsäule zugleich als Beitrag zur Physiologie des menschlichen Rückenmarks, Mitt. Grenzgeb. Med. Chir. I (1896) 415-660, S. 546.

${ }^{11}$ Siehe de Quervain, $\mathrm{F}$., Les traumatismes du rachis, rapport principal in: $I I^{e}$ Congrès de la Société Internationale de Chirurgie 1908, Bruxelles 1908, S. 687-734.

${ }^{12}$ Head, H., and T.Thompson, The grouping of afferent impulses within the spinal cord, Brain 29 (1906) S. 37-741, S. 603.

${ }^{13}$ de Quervain, F., Die Entwicklung der Chirurgie des Nervensystems in der Schweiz, in Festschrift zum fünfundzwanzigjährigen Bestehen der schweizerischen Gesellschaft für Chirurgie, Basel 1939, S.67-76, S.69.

${ }^{14}$ Kocher, op. cit., Anm. 10.

15 Ibid. S. 657.

${ }^{16}$ Starr, A., Local anaesthesis as a guide to the diagnosis of lesions of the upper portion of the spinal cord, Brain 17 (1894) 481-514.

${ }_{17}$ Siehe z. B. das Lehrbuch von H.Oppenheim, Textbook of nervous diseases (englische Ausgabe), Edinburgh 1911, S.125-136, 261-273, und O.Foerster's klassische Arbeit: Symptomatologie der Erkrankungen des Rückenmarks und seiner Wurzeln, in: Bumke, O., und O. Foerster, Handbuch der Neurologie, Berlin 1936, Vol. V, S. 173.

${ }^{18}$ Ibid. Die Originalstelle ist bei Kocher, op. cit. Anm.10, S. 556: Einseitige Kontraktion der unteren Abdominalmuskulatur bei Kompression der Testes.

${ }^{19}$ Head, H., and A. W. Campbell, The pathology of Herpes Zoster and the bearing on sensory localization, Brain 23 (1900) 353-523.

${ }^{20}$ Siehe de Quervain, op. cit., Anm. 10.

${ }^{21}$ Foerster, op. cit., Anm. 17, S. 249.

${ }^{22}$ Behan, R.J., Pain, its origin, conduction, perception and diagnostic significance, New York/London, 1915, S.55.

${ }^{23}$ Sahli, H., Lehrbuch der klinischen Untersuchungsmethoden, Leipzig/Wien, erstmals in der 2. Aufl. 1894, S. 860-65; ibid. 7. Aufl., 1932, Vol. III, S. 658, 661 f., 665-671.

Osler, W., The principles and practice of medicine, London/New York. 6. Aufl. 1905, S. 878-879 (Tafeln) und 880 (Text), unverändert in der 15. Aufl. 1944, S. 1222-1226.

${ }^{24}$ de Quervain, F., Spezielle chirurgische Diagnostik, Leipzig, 1. deutsche Aufl. 1907, S.390-396; die 10. deutsche Aufl. 1950, S.486f., enthält nur die spinalen Motilitätstafeln. 
25 Nissen, R., Der Beitrag der Schweiz zur modernen Chirurgie; in: Blaser, R. und H. Buess (Hrsg.), Aktuelle Probleme in der Geschichte der Medizin, Basel/New York 1966, S.445-451, S. 449 .

${ }_{26}$ Faber, K., Nosography, 2. Aufl., New York 1930, S.28-58 (Neudruck 1978).

27 Ibid., siehe auch Lain Entralgo, P., La Historia clinica, Madrid 1950.

28 Müller, L. A., Die topographischen Beziehungen des Hirns zum Schädel, Diss. med. Bern 1889.

${ }^{29}$ Kocher, T., Chirurgische Operationslehre, 1. Aufl., Jena 1892, S.31-38; ibid. 3. Aufl. 1897, S. $51-58$.

siehe auch de Quervain, F., La Suisse, in: Chipault, A. (Hrsg.), L'état actuel de la chirurgie nerveuse, Paris 1902, Vol. I, 756-793, S. 758.

${ }^{30}$ Kocher, ibid., 1.Aufl. S. 36.

31 Ibid., 5. Aufl. 1907, S.291-292.

32 Ibid., 2. Aufl. 1894, S.2.

33 Wien 1901, S. 408-424.

${ }^{34}$ Ibid., S. 403-404.

${ }^{35}$ Ibid., S.423; Madritsch, W., Der Zürcher Chirurg Rudolf Ulrich Krönlein, Diss. med. Zürich 1967, S. 33 .

${ }^{36}$ Cushing, H., Surgery of the Head, in: Keen, W. W., Surgery, its Principles and Practice, Philadelphia/London 1908, Vol. III, 17-276, S. 168-172.

37 Siehe den Rückblick von Richter, H., Vereinigung Schweizer Neurochirurgen, Swiss Med.4. (1982), 9, 15-18.

${ }^{38}$ Kochers Beitrag zur Dermatom-Forschung ist anscheinend auch von Historikern übersehen worden. Er findet keine Erwähnung in der langen historischen Einleitung zu J.F. Fultons Physiology of the nervous system, London/Toronto 1938, noch in Garrinsons klassischer History of Neurology, rev. by L.C.McHenry, Springfield 1969, noch in E.Clarke and C. D. O'Malley's großer Geschichte The human brain and spinal cord, Berkeley/Los Angeles 1968.

\section{Summary}

Around 1900 the Swiss Nobel Prize winner Theodor Kocher (1841-1917) was one of a few clinicians aiming at preoperative topographic diagnosis of pathological changes in the central nervous system. Thus on the basis of the correlation of neurological symptoms with local lesions verified at operation or autopsy Kocher worked out a cranio-cerebral topography. More important, he further corroborated the concept of spinal dermatomes in man which had been put forward earlier, especially by the animal work of Charles S. Sherrington. Indeed, Kocher deserves credit for the first complete chart of human dermatomes (1896) both sensory and motor-antedating that of Henry Head by four years. This work which had immediate repercussions both in clinical medicine and physiology was internationally recognized until World War II and the subsequent development of neuroradiology.

PD Dr.med. et phil. Ulrich Tröhler

Medizinhistorische Bibliothek, Klingelbergstraße 23, CH-4031 Basel 